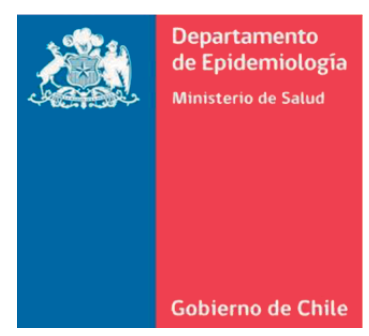

\title{
Protocolo de manejo simplificado para pacientes con hepatitis C crónica. Chile, 2019
}

\author{
Subsecretaría de Salud Pública \\ División de Prevención y Control de Enfermedades \\ Departamento de Enfermedades Transmisibles
}

\section{Contenido}

1. Introducción

2. Objetivo

3. Usuarios a los que está dirigido

4. Declaración de intenciones

5. Epidemiología e historia natural

6. Diagnóstico y tamizaje

7. Evaluación inicial

8. Algoritmo de terapia

9. Tratamiento en pacientes con insuficiencia renal

10. Tratamiento en pacientes con falla a terapia previa

11. Seguimiento post-tratamiento

12. Responsables

13. Equipo de Trabajo

14. Revisión interna y externa

15. Declaración de conflictos de interés

16. Referencias ca (reducir incidencia en $90 \%$ y mortalidad en $65 \%)^{\mathrm{i}}$ por otro lado, son los factores que hacen necesario contar con un algoritmo simplificado de manejo actualizado de la enfermedad. Este documento pretende ser un recurso que guíe las decisiones terapéuticas en el manejo de esta enfermedad.

\section{Objetivo}

Contar con un protocolo simplificado del manejo clínico del paciente adulto con hepatitis $\mathrm{C}$, cuyo tratamiento involucra antivirales de acción directa (AAD) pangenotipos. Este protocolo permite facilitar la atención de los pacientes, así como el uso eficiente de los recursos.

\section{Usuarios a los que está dirigido}

Este documento está dirigido a los equipos tratantes, médicos y enfermeras, que atienden directamente al paciente con hepatitis $\mathrm{C}$ crónica. Además permite dar guía a los equipos externos que participan directa o indirectamente con los pacientes, como tecnólogos médicos, químicos farmacéuticos, referentes de los Servicios de Salud y tomadores de decisiones.

\section{Declaración de intenciones}

Este protocolo fue elaborado con la intención de establecer estándares generales de cuidado para pacientes, pero las decisiones individuales para cada persona sólo pueden ser determinadas por profesionales competentes sobre la base de toda la información clínica respecto del caso. Las recomendaciones entregadas están sujetas a modificaciones conforme

\footnotetext{
i Organización Mundial de la Salud. Estrategia Mundial del
} sector de la salud contra las Hepatitis víricas. 2016-2021. 
al avance del conocimiento científico, las tecnologías disponibles en cada contexto en particular, y según evolucionan los patrones de atención.

Este protocolo se ha elaborado debido a la necesidad de contar con un recurso de manejo clínico a los equipos tratantes, complementando la guía clínica GES por metodología GRADE que se encuentra en desarrollo, cuya entrega está estimada para el año 2020 .

\section{Epidemiología e historia natural}

De acuerdo a estimaciones de la Organización Mundial de la Salud, en 2015 la prevalencia mundial de infección por el virus de la hepatitis C (VHC) era de $1 \%$ (aproximadamente 71 millones de personas) ${ }^{1,2}$. La transmisión del virus es por vía parenteral, incluyendo transfusión de productos sanguíneos, prácticas de inyección no seguras (incluyendo transmisión asociada a los cuidados de la salud y transmisión entre usuarios de drogas intravenosas) $)^{3,4}$. Menos frecuentemente la transmisión puede ser por vía vertical de la madre infectada al hijo y por vía sexual, especialmente en el contexto de hombres que tienen sexo con hombres, con o sin co-infección por VIH.

El virus de la hepatitis $C$ tiene diferentes variantes (genotipos). En nuestro país el genotipo más frecuente es el $1 \mathrm{~b}$ (80\% aproximadamente), seguido por el genotipo $3(18 \%)^{5}$. Los genotipos tienen importancia en el entendimiento de la epidemiología y durante mucho tiempo fueron determinantes en la elección de las terapias, sin embargo, en la medida que se cuenta con regímenes terapéuticos pangenotípicos, la necesidad de su determinación en la práctica clínica podría disminuir.

En Chile se estima que existen alrededor de 35.000 personas infectadas, de las cuales están diagnosticadas menos de 10.000. Dado que la infección es frecuentemente asintomática, es relevante fortalecer las estrategias de diagnóstico y tamizaje efectivas.

El VHC puede producir hepatitis tanto aguda como crónica. En la etapa aguda se detectan síntomas sólo en un $20 \%$ de los afectados. Entre 15 y $45 \%$ de los infectados logran eliminar la infección en forma espontánea, habitualmente dentro de los primeros 6 meses desde la infección. El 55 a 85\% restante de los pacientes desarrollan una infección crónica, en la que se produce inflamación y fibrosis hepática progresiva. Esta etapa, si bien es frecuentemente asintomática, puede acompañarse de manifestaciones extrahepáticas $^{6}$. Las más frecuentes son crioglobulinemia (30\%), depresión (24\%), diabetes mellitus (15\%) y enfermedad renal $(10 \%)$. Es destacable que estas manifestaciones pueden ser modificadas por el tratamiento antiviral. La progresión a cirrosis ocurre en un 15 a
$30 \%$ de los pacientes a los 20 años de la infección. La etapa de cirrosis compensada puede complicarse por descompensación (ascitis, hemorragia variceal o encefalopatía hepática) en forma progresiva o por desarrollo de carcinoma hepatocelular, lo que ocurre con una frecuencia de 1 a $3 \%$ al año.

\section{Diagnóstico y tamizaje}

El diagnóstico de la infección por VHC se debe sospechar ante la presencia de factores de riesgo de contacto parenteral. Dado que la infección es frecuentemente asintomática y que hasta el $43 \%$ de los infectados en nuestro medio no tienen un factor de riesgo identificado ${ }^{5}$ (, en la actualidad se recomienda agregar estrategias de tamizaje en determinadas cohortes de edad de mayor riesgo. De este modo, los grupos en que se recomienda realizar anticuerpos contra VHC son:

- Todas las personas mayores de 45 años una vez en la vida.

- Personas que hayan recibido una transfusión de hemoderivados o trasplante de órganos antes de 1996.

- Personas que hayan usado o sean usuarias de drogas intravenosas.

- Pacientes hemofílicos expuestos a hemoderivados.

- Pacientes sometidos a diálisis.

- Pacientes con elevación persistente de aminotransferasas.

- Pacientes con cirrosis hepática de cualquier etiología.

- Pacientes infectados con VIH/SIDA.

- Niños nacidos de una madre infectada con VHC.

- Personal de salud expuesto a sangre infectada por vía percutánea o mucosa.

- Parejas sexuales de pacientes infectados.

Todos los resultados positivos de anti-VHC deben confirmarse mediante la detección de RNA viral en sangre, lo que puede realizarse con exámenes cualitativos o cuantitativos. Estos deben estar validados y tener una sensibilidad mínima de $50 \mathrm{IU} / \mathrm{mL}$.

Por norma, todos los resultados positivos locales de anticuerpos para $\mathrm{VHC}^{\mathrm{ii}, \mathrm{iii}}$ son enviados al Instituto de Salud Pública (ISP) para su confirmación.

Debido a que en el informe del ISP se cuenta con el resultado del examen con la determinación de

ii Circular $\mathrm{N}^{\mathrm{o}}$ B 21/28. 12 de noviembre de 2008.- Instruye a todos los centros y bancos de sangre de la red asistencial de salud sobre casos confirmados de Hepatitis C enviar muestra de confirmación al ISP.

iii Ordinario 0068, del 17 de enero de 2012.-Prestaciones de confirmación exentas de pago de arancel público y privado en el ISP. 
RNA cualitativo, no es necesario volver a solicitar PCR confirmatoria del laboratorio local. Es relevante considerar que en el reporte del ISP lo que confirma la infección activa (con viremia) es la positividad del Ampli Prep Cobas Taqman HCV ${ }^{\text {iv }}$.

No es necesario realizar genotipificación de VHC en la mayoría de los pacientes, ya que la terapia de elección es pangenotípica.

\section{Evaluación inicial}

En los pacientes con diagnóstico confirmado se requiere evaluar los siguientes aspectos:

\section{a) Medidas generales}

- Evaluar consumo de alcohol y aconsejar su suspensión.

- Manejo de la obesidad.

- Chequeo a contactos epidemiológicos ${ }^{\mathrm{v}}$ (pareja sexual, hijos en caso de ser mujer).

- Educación en la prevención de transmisión: No compartir hojas de afeitar, evitar conductas sexuales de riesgo, reducir riesgo en personas que usan drogas intravenosas.

- Determinación de AgsHB, anti-HBc total y/o anti-HBs, y anti-VHA IgG para decidir vacuna de hepatitis A y B.

- ELISA VIH.

- Descartar embarazo en mujeres en edad fértil.

\section{b) Determinación de la etapa de fibrosis hepática}

- La evaluación de la fibrosis hepática (particularmente la detección de fibrosis avanzada/cirrosis) es relevante para el pronóstico y seguimiento del paciente, pero no es estrictamente necesaria para el inicio de la terapia antiviral en la mayoría de los pacientes.

- En muchos pacientes la evaluación clínica: estigmas de cirrosis al examen físico, imágenes hepáticas sugerentes de daño hepático crónico, esplenomegalia, trombocitopenia o historia de descompensaciones de cirrosis son suficientes para hacer el diagnóstico de cirrosis.

- Se recomienda la evaluación no invasiva de fibrosis hepática mediante la combinación de métodos serológicos con métodos físicos (elastografía).

- Métodos serológicos:

\footnotetext{
${ }^{\text {iv }}$ Dado que algunas personas tienen infección que aclara espontáneamente, podría haber anti-HCV positivo, Inno Lia positivo y Taqman negativo (con resultado del ISP positivo), pero que no tienen infección y por lo tanto no requieren tratamiento.

${ }^{v}$ Circular B51 No 13 del 27/11/2017. Circular epidemiológica der Hepatitis B y C.
}

i. APRI ${ }^{\mathrm{vi}}$. Un valor APRI $<0,5$ descarta cirrosis razonablemente y un valor $>2$ es sugerente de cirrosis (especificidad 91\%, sensibilidad 46\%).

ii. FIB-4 ${ }^{\text {vii }}$. Un valor de FIB-4 $<1,45$ descarta cirrosis razonablemente y un valor $>3,25$ es sugerente de cirrosis.

iii.Se recomienda realizar elastometría hepática con el método más validado (Elastografía). Valores de rigidez hepática $>10 \mathrm{kPa}$ son sugerentes de fibrosis significativa (F3) y valores $>13 \mathrm{kPa}$ sugieren cirrosis (F4).

- La biopsia hepática sólo está recomendada en aquellos pacientes con duda razonable de la etapa de fibrosis hepática y resultados discordantes entre los métodos serológicos y la elastografía.

\section{c) Evaluación general del paciente con cirrosis}

- Evaluar si el paciente es potencial candidato a trasplante. En general, cualquier paciente menor de 70 años, Child-Pugh B o C o MELD > 15 sin comorbilidades significativas debe ser derivado para evaluación de trasplante hepático.

- Tamizaje de carcinoma hepatocelular con ecografía abdominal y alfafetoproteína (AFP) cada 6 meses.

- Tamizaje de várices esofágicas con endoscopia digestiva alta.

- Vacuna neumococo.

- Vacuna influenza cada año.

- Vacuna hepatitis $\mathrm{A}^{\text {viii }}$ y B según serología (ver punto a).

- Identificar pacientes con cirrosis descompensada (Child-Pugh B o C) o con antecedente previo de descompensación. Estos pacientes no deben recibir inhibidores de proteasa y en su tratamiento se debe incluir el uso de ribavirina (ver algoritmo de terapia).

\section{d) Evaluación de función renal}

- En pacientes con depuración de creatinina $<30$ $\mathrm{mL} / \mathrm{min}$ no debe usarse regímenes basados en sofosbuvir ${ }^{\mathrm{ix}}$.

e) Evaluar potenciales interacciones farmacológicas

- Se deben examinar el listado de medicamentos que el paciente está recibiendo y revisar posibles interacciones medicamentosas. Se recomienda usar el sitio de la Universidad de Liverpool hepdruginteractions.org para este efecto.

\footnotetext{
${ }^{v i}$ https://www.hepatitisc.uw.edu/page/clinical-calculators/apri

vii $\mathrm{https} / / /$ www.hepatitisc.uw.edu/page/clinical-calculators/fib-4

viii Exento No97 del 20/08/2018. Incluye vacuna antihepatitis A a pacientes con Hepatitis C. 2 Dosis 0 y 6 meses.

ix Esto podría cambiar en la medida que se tenga más información de estudios que están utilizando sofosbuvir en pacientes con falla renal en diálisis.
} 


\section{Algoritmo de terapia}

Se recomienda el tratamiento a todas las personas infectadas mayores de 12 años en que haya una expectativa de vida mayor de 6 meses, independientemente de la etapa de la enfermedad. Con el objetivo de simplificar al máximo el esquema de tratamiento (de acuerdo a la recomendación de la $\mathrm{OMS}^{7}$ ), se propone utilizar una terapia pangenotípica y "panfibrótica", vale decir esquema de tratamiento y duración es la misma para la mayoría de los pacientes independiente del genotipo y de la etapa de fibrosis. Por esta razón, se ha privilegiado el esquema de terapia con sofosbuvir/velpatasvir (SOF/VEL) por 12 semanas $^{8,9}$. (Incluido en el GES).

En cuanto al manejo práctico de esta terapia, se debe recordar:

- La dosis es un comprimido al día de sofosbuvir $400 \mathrm{mg}+$ velpatasvir $100 \mathrm{mg}$ (tableta combinada) por 12 semanas.

- Puede administrarse con o sin comidas.

- El medicamento puede almacenarse a temperatura ambiente.

- Se deben revisar interacciones farmacológicas (hep-druginteractions.org). Hay que recordar que el uso de amiodarona es una contraindicación absoluta por el riesgo de arritmias.

- No debe utilizarse inhibidores de bomba de protones en dosis altas o en 2 dosis al día. Si se utilizara omeprazol o similares, deben administrarse una vez al día 4 h después de la dosis de sofosbuvir/ velpatasvir.
- Se recomienda realizar un seguimiento durante el tratamiento que permita optimizar adherencia, sin embargo, no es necesario el control de carga viral durante el tratamiento ni exámenes frecuentes.

- En pacientes con cirrosis descompensada o antecedente de descompensación se debe agregar ribavirina $^{10}$ en dosis de $1.200 \mathrm{mg}$ (partir con 600 $\mathrm{mg}$ al día, aumentar gradual según tolerancia y hemograma). Este grupo de pacientes debe ser seguido frecuentemente (cada 2 a 4 semanas) e idealmente seguido en conjunto con hepatólogo.

- La terapia en pacientes co-infectados con VIH es $\operatorname{similar}^{11}$ (Figura 1).

En pacientes con situaciones especiales no descritas en este algoritmo, por ejemplo, genotipo 3 con descompensación, insuficiencia renal con cirrosis descompensada, falla a tratamiento con antivirales de acción directa, se recomienda interconsultar a equipo de hepatología o al Comité Asesor de Hepatitis Viral del Ministerio.

\section{Tratamiento en pacientes con insuficiencia renal}

Los pacientes con insuficiencia renal leve a moderada (depuración de creatinina $>30 \mathrm{~mL} / \mathrm{min}$ ) deben recibir el tratamiento estándar (ver punto 4).

Los pacientes con insuficiencia renal etapa 4 y 5 (depuración de creatinina $<30 \mathrm{~mL} / \mathrm{min}$ ) no deben recibir terapias basadas en sofosbuvir, en su lugar, se pueden utilizar dos opciones:
Figura 1. Esquema de tratamiento simplificado de hepatitis $C$ con terapia pangenotípica (EDA: Endoscopia Digestiva alta).

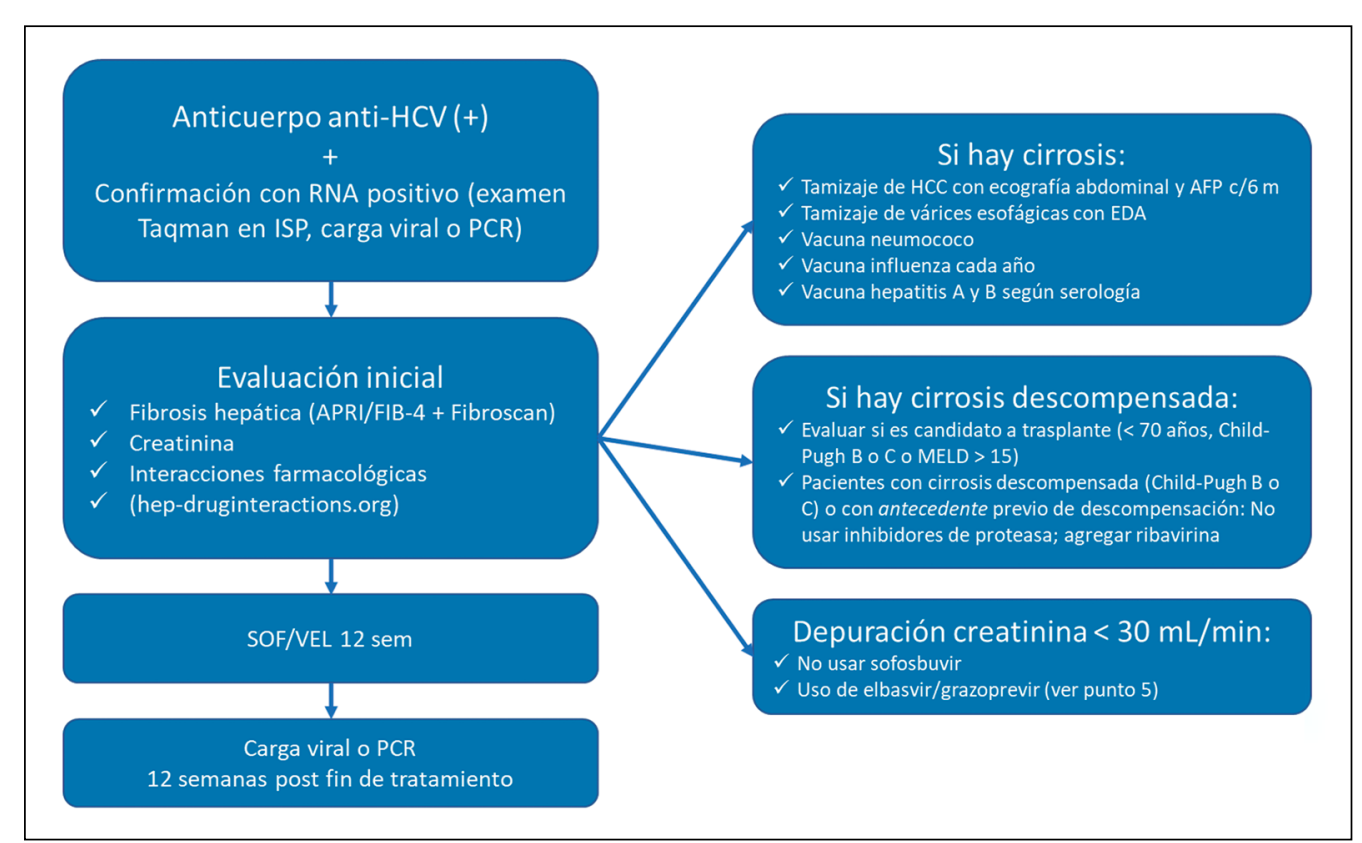


- Elbasvir/grazoprevir ${ }^{12}$ : Sólo en genotipo 1 y 4 (Incluido en el GES).

- Glecaprevir/pibrentasvir ${ }^{13}$ : Pangenotípico. (Actualmente la terapia no cuenta con Registro sanitario del Instituto de Salud Pública, por lo que no está disponible en el país).

La duración de la terapia es de 12 semanas para ambos esquemas.

\section{Tratamiento en pacientes con falla a terapia previa}

En los pacientes no respondedores a terapias con antivirales de acción directa se recomienda realizar genotipificación de VHC y revisar la adherencia a la terapia e interacciones farmacológicas.

La terapia ideal de rescate en estas situaciones es el uso sofosbuvir/velpatasvir/voxilaprevir ${ }^{14,15}$. Actualmente esta terapia cuenta con Registro sanitario del ISP, pero no está disponible dentro de las prestaciones del GES 2019-2022.

En casos en que no sea prudente esperar, se recomienda contactar a la unidad de hepatología o al Comité Asesor de Hepatitis Viral del Ministerio (hepatitis@minsal.cl) para analizar el caso y sugerir una estrategia de manejo.

\section{Seguimiento post-tratamiento}

La respuesta viral sostenida (RVS) se define como una carga viral indetectable o un PCR cualitativo negativo de VHC 12 semanas luego del fin de tratamiento. La RVS es habitualmente mantenida de por vida, en estudios de seguimiento por varios años, sólo un $0,5 \%$ de los pacientes tuvieron una recaída, siempre dentro del primer año. Por lo anterior, no se recomienda realizar cargas virales adicionales luego de lograda la RVS en la mayoría de los pacientes.

Dado que la infección por VHC no deja inmunidad permanente, los pacientes pueden reinfectarse si se exponen nuevamente al virus. Se recomienda, por lo tanto, realizar un PCR o carga viral una vez al año en pacientes con factores de riesgo persistentes de infección (usuarios de drogas intravenosas y hombres que tienen sexo con hombres).

En pacientes con fibrosis avanzada, definida como F3 y F4 de la escala de METAVIR se recomienda lo siguiente ${ }^{16}$ :

- Vigilancia de carcinoma hepatocelular de por vida cada 6 meses con ecografía abdominal y AFP.

- Tamizaje de várices esofágicas. Si no hay várices esofágicas en 2-3 años, puede suspenderse el tamizaje.
- El seguimiento con elastografía es razonable, sin embargo, una regresión de la fibrosis por esta técnica no permite suspender el tamizaje de hepatocarcinoma (HCC).

El Decreto No22 del 01/07/2019, con el listado de prestaciones (LEP) de esta patología, se puede encontrar en la página web minsal.cl.

\section{Responsables de la División de Control y Prevención de Enfermedades}

Dra. Sylvia Santander Rigollet

Jefa de la División de Prevención y Control de

Enfermedades

Subsecretaría de Salud Pública

Ministerio de Salud

Patricia Contreras Cerda

Jefa (S) Departamento de Enfermedades

Transmisibles

División de Prevención y Control

Subsecretaria de Salud Pública

Ministerio de Salud

Andrea Peña Otárola

Referente Nacional Programa Hepatitis B y C

Departamento de Enfermedades Transmisibles

División de Prevención y Control de Enfermedades

Subsecretaría de Salud Pública

Ministerio de Salud

\section{Equipo Técnico de Trabajo}

Dr. Alejandro Soza Ried

Gastroenterólogo de Adultos

Hepatólogo

Profesor Asociado de Medicina

Pontificia Universidad Católica de Chile

Past- Presidente. Asociación Chilena de Hepatología Integrante del Comité Asesor de Hepatitis virales. MINSAL

Andrea Peña Otárola

Referente Nacional Programa Hepatitis B y C

Departamento de Enfermedades Transmisibles

División de Prevención y Control de Enfermedades

Subsecretaría de Salud Pública

\section{Revisión interna y externa}

Mario Soto Marchant

Cristián Ramírez

Departamento de Epidemiología

División de Planificación Sanitaria

Subsecretaría de Salud Pública

Ministerio de Salud 
María Fernanda Espinoza Soto

Enfermera Jefe del Servicio de Cardiología,

Gastroenterología y Anestesia

Enfermera especializada y responsable del Programa

de Hepatitis C

Hospital Barros Luco Trudeau

Dr. Gabriel Mezzano Puentes

Médico Gastroenterólogo-Hospital del Salvador-

Clínica Universidad de los Andes

Profesor asociado Universidad de Chile

Integrante del Comité Asesor de Hepatitis del

Ministerio de Salud

Dra. Leyla Nazal Ortiz

Médico Gastroenteróloga

Clínica Las Condes

Presidenta Asociación Chilena de Hepatología

Dra. Pamela Yaquich Saud

Médico Gastroenteróloga

Hospital San Juan de Dios

Jorge Robinovich T.

Guillermo Mundt V.

Fundación de Pacientes con Hepatitis (FUPAHEP)

Se solicitó la revisión del comité asesor del Ministerio de Salud, la Asociación Chilena de Hepatología, Fundación de Pacientes con Hepatitis, Departamento GES y Redes de Alta Complejidad, Depto. de Procesos Clínicos y Gestión Hospitalaria, ambos de la División de Gestión de la Red Asistencial.

\section{Declaración de conflictos de interés}

El Dr. Soza declara conflictos de interés por lo que se realizó una revisión del documento por el comité asesor de Hepatitis B y C, perteneciente al Ministerio de Salud de Chile, quedando conformes con las recomendaciones entregadas.

\section{Referencias}

1.- Polaris Observatory HCV Collaborators. Global prevalence and genotype distribution of hepatitis $\mathrm{C}$ virus infection in 2015: a modelling study. Lancet Gastroenterol Hepatol 2017;2:161-76.

2.- World Health Organization. Global Hepatitis Report 2017. World Health Organization; 2017.

3.- Pépin J, Abou Chakra CN, Pépin E, Nault V, Valiquette L. Evolution of the global burden of viral infections from unsafe medical injections, 2000-2010. PLoS ONE 2014; 9:e99677.

4.- Prati D. Transmission of hepatitis C virus by blood transfusions and other medical procedures: a global review. J Hepatol 2006;45:607-16.
5.- Soza A, Arrese M, González R, Álvarez M, Pérez RM, Cortés $\mathrm{P}$, et al. Clinical and epidemiological features of 147 Chilean patients with chronic hepatitis C. Ann Hepatol 2004;3(4):146-51.

6.- Younossi ZM, Birerdinc A, Henry L. Hepatitis C infection: A multi-faceted systemic disease with clinical, patient reported and economic consequences. J Hepatol 2016;65(1 Suppl):S109-S119.

7.- Organización Panamericana de la Salud. Directrices para la atención y el tratamiento de las personas diagnosticadas de infeccióncrónica por el virus de la hepatitis C. World Health Organization; 2018.

8.- Feld JJ, Jacobson IM, Hézode C, Asselah T, Ruane PJ, Gruener N, et al. Sofosbuvir and velpatasvir for HCV genotype 1, 2, 4, 5, and 6 infection. N Engl J Med 2015; 373:2599-607.

9.- Foster GR, Afdhal N, Roberts SK, Bräu N, Gane EJ, Pianko S, et al. Sofosbuvir and velpatasvir for HCV genotype 2 and 3 infection. N Engl J Med 2015;373: 2608-17.

10.- Curry MP, O’Leary JG, Bzowej N, Muir AJ, Korenblat KM, Fenkel JM, et al. Sofosbuvir and Velpatasvir for HCV in Patients with Decompensated Cirrhosis. N Engl J Med 2015;373:2618-28.

11.- Wyles D, Bräu N, Kottilil S, Daar ES, Ruane P, Workowski K, et al. Sofosbuvir and Velpatasvir for the Treatment of Hepatitis C Virus in Patients Coinfected With Human Immunodeficiency Virus Type 1: An OpenLabel, Phase 3 Study. Clin Infect Dis 2017;65:6-12.

12.- Roth D, Nelson DR, Bruchfeld A, Liapakis A, Silva M, Monsour H, et al. Grazoprevir plus elbasvir in treatmentnaive and treatment-experienced patients with hepatitis $\mathrm{C}$ virus genotype 1 infection and stage 4-5 chronic kidney disease (the C-SURFER study): a combination phase 3 study. The Lancet 2015;386:1537-45.

13.- Gane E, Lawitz E, Pugatch D, Papatheodoridis G, Bräu N, Brown A, et al. Glecaprevir and Pibrentasvir in Patients with HCV and Severe Renal Impairment. N Engl J Med 2017;377(15):1448-55.

14.- Bourlière M, Gordon SC, Flamm SL, Cooper CL, Ramji A, Tong M, et al. Sofosbuvir, velpatasvir, and voxilaprevir for previously treated $\mathrm{HCV}$ infection. $\mathrm{N}$ Engl J Med 2017;376:2134-46.

15.- Bourlière M, Gordon SC, Schiff ER, Tran TT, Ravendhran N, Landis CS, et al. Deferred treatment with sofosbuvir-velpatasvir-voxilaprevir for patients with chronic hepatitis $\mathrm{C}$ virus who were previously treated with an NS5A inhibitor: an open-label substudy of POLARIS-1. Lancet Gastroenterol Hepatol 2018;3:55965.

16.- Jacobson IM, Lim JK, Fried MW. American Gastroenterological Association Institute Clinical Practice Update-Expert Review: Care of Patients Who Have Achieved a Sustained Virologic Response After Antiviral Therapy for Chronic Hepatitis C Infection. Gastroenterology 2017;152:1578-87. 